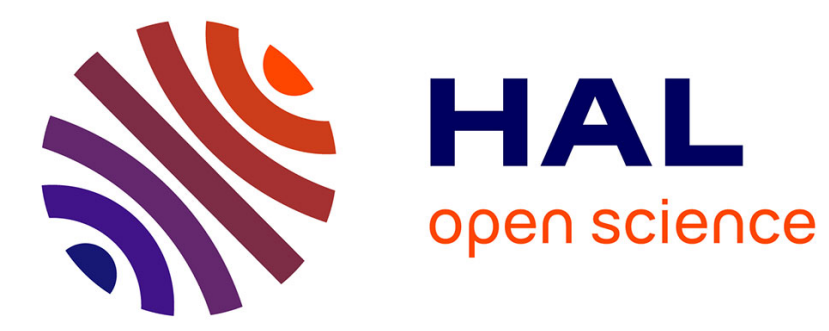

\title{
Diffusion of a chain : concentration effects
}

M. Daoud, G. Jannink

\section{To cite this version:}

M. Daoud, G. Jannink. Diffusion of a chain : concentration effects. Journal de Physique Lettres, 1980, 41 (9), pp.217-220. 10.1051/jphyslet:01980004109021700 . jpa-00231763

\section{HAL Id: jpa-00231763 https://hal.science/jpa-00231763}

Submitted on 1 Jan 1980

HAL is a multi-disciplinary open access archive for the deposit and dissemination of scientific research documents, whether they are published or not. The documents may come from teaching and research institutions in France or abroad, or from public or private research centers.
L'archive ouverte pluridisciplinaire HAL, est destinée au dépôt et à la diffusion de documents scientifiques de niveau recherche, publiés ou non, émanant des établissements d'enseignement et de recherche français ou étrangers, des laboratoires publics ou privés. 


\title{
Diffusion of a chain : concentration effects
}

\author{
M. Daoud \\ Boston University, Physics Department, Boston, MA 02215, U.S.A. \\ and G. Jannink
}

C.E.N. Saclay, S.P.S.R.M., B.P. No 2, 91190 Gif-sur-Yvette, France

(Rę̧u le 12 avril 1979, révisé le 6 février, accepté le 5 mars 1980)

\begin{abstract}
Résumé. - La concentration de cross-over entre solutions diluées et semi-diluées est la même pour les propriétés dynamiques que pour les propriétés statiques de polymères flexibles en bon solvant. En solution semi-diluée, l'exposant $\beta$ dans la variation du coefficient de diffusion du blob en fonction de la concentration en monomères $D_{c} \sim C^{\beta}$ varie avec la température et la concentration. La variation de cet exposant effectif n'est pas monotone. Cet effet peut se vérifier en comparant les résultats des expériences de diffusion élastique des neutrons et quasiélastique de la lumière.

Abstract. - It is shown that the cross-over concentration between dilute and semi-dilute solutions is the same for dynamical properties as for static properties of flexible polymers in a good solvent. For semi-dilute solutions, the exponent $\beta$ in the concentration dependence of the diffusion coefficient of a blob $D_{\mathrm{c}} \sim C^{\beta}$ is expected to depend on both temperature and concentration. The variation of this effective exponent is non monotonous. A combined neutron elastic and hight quasielastic scattering experiment is proposed to observe this effect.
\end{abstract}

Recently, Weill and des Cloizeaux [1], Akcasu and Han [2] (A.H.W.C.) have proposed a model for the dynamics of a flexible polymer chain in a good solvent [3]. This approach, based on the blob model [10], shows that the cross-over effects between theta and good solvents are much more important for the dynamical properties, where one has to calculate mean values of inverse distances than for the radius of gyration for instance, where $\left\langle r^{2}\right\rangle$ is calculated. As a result, the experimental studies [4] measure an effective exponent, as was suggested by Stockmayer and Albrecht [5] some time ago.

In this note, we wish to clarify the cross-over between dilute and semi-dilute solutions in a good solvent, and extend the A.H.W.C. approach to semidilute solutions.

The cross-over concentration $C^{*}$ is usually defined via the (static) radius of gyration $R_{\mathrm{G}}$ as [10]

$$
C^{*} \sim \frac{N}{R_{\mathrm{G}}^{3}} \sim N^{1-3 v} \approx N^{-4 / 5}
$$

where $N$ is the number of statistical units in the chain, $C$ the monomer concentration, and $v$ the excluded volume exponent [3], [6].
If we consider now the diffusion coefficient $D_{0}$ of a chain, or the viscosity of a dilute solution, we have

$$
D_{0} \sim \frac{1}{R_{\mathrm{H}}} \sim \frac{1}{N^{v^{\prime}}}
$$

where $R_{\mathrm{H}}$ is called the hydrodynamic radius [7] and where the effective exponent [1], [2] $v^{\prime}$ is less than $v$ [4] (usually $v^{\prime} \approx 0.54$ )

$$
\eta=\eta_{\mathrm{s}}\left(1+[\eta] C+K_{\mathrm{H}}[\eta]^{2} C^{2}+\cdots\right)
$$

where $\eta_{\mathrm{s}}$ is the viscosity of the solvent, $[\eta]$ the intrinsic viscosity [7] and $K_{\mathrm{H}}$ the Huggins coefficient, which is a constant [3], [8]. It can be shown that [9]

$$
[\eta] \sim \frac{T}{N} \sim \frac{R_{\mathrm{G}}^{2} R_{\mathrm{H}}}{N} \sim N^{2 v+v^{\prime}-1} .
$$

A straight forward (but wrong) dimensional analysis would then lead to different laws for the cross-over between dilute and semi-dilute solutions, namely

$$
\begin{aligned}
& C_{\mathbf{D}}^{*} \sim N^{1-3 v^{\prime}}=N^{-0.62} \\
& C_{\eta}^{*} \sim[\eta]^{-1} \sim N^{1-2 v-v^{\prime}}=N^{-0.74} .
\end{aligned}
$$


The flaw in the analysis lies in the fact that there is not only one, but two lengths [10] in the problem (none of them being $R_{\mathrm{H}}$ ). $R_{\mathrm{H}}$ cannot be considered as a characteristic length, except in the asymptotic region of very high $N$, which according to refs. [1] and [2] seems impossible to reach in the present state of the art. In order to calculate $R_{\mathrm{H}}$, [1], [2] one has to separate short from large distances [10] namely

$$
\begin{gathered}
R_{\mathrm{H}}^{-1}=\frac{1}{2 N^{2}} \sum_{i, j}\left\langle r_{i j}^{-1}\right\rangle \\
r_{i j} \sim \begin{cases}|i-j|^{1 / 2} & \text { if } 1<|i-j| \leqslant N_{\tau} \\
\left(\frac{|i-j|}{N_{\tau}}\right)^{v} N_{\tau}^{1 / 2} & \text { if } \quad N_{\tau}<|i-j|<N\end{cases}
\end{gathered}
$$

and [8], [2]

$$
N_{\tau} \sim\left(\frac{T-\Theta}{\Theta}\right)^{-2} \equiv \tau^{-2} .
$$

We may call the substructure associated to $N_{\tau}$ a flof.

This analysis [1], [2], valid for dilute solutions, can be extended to semi-dilute solutions, as shown, below. So all the following concerns semi-dilute solutions.

Let us consider the diffusion coefficient, which is more appropriate, as relation (3) does not hold for high concentrations [11]. In semi-dilute solutions, another substructure, called the blob [10], appears. It is associated to a number of monomers $g \geqslant N_{\tau}$, and to an average squared length $\xi^{2}$ between successive contacts. We know that inside a blob (i.e. for short distances) the segment has a single chain behaviour [12]. Thus the blob may be considered as a single chain, with a flof substructure identical to that of a single chain [15] (see Fig. 1). This substructure has not been directly checked experimentally, but we
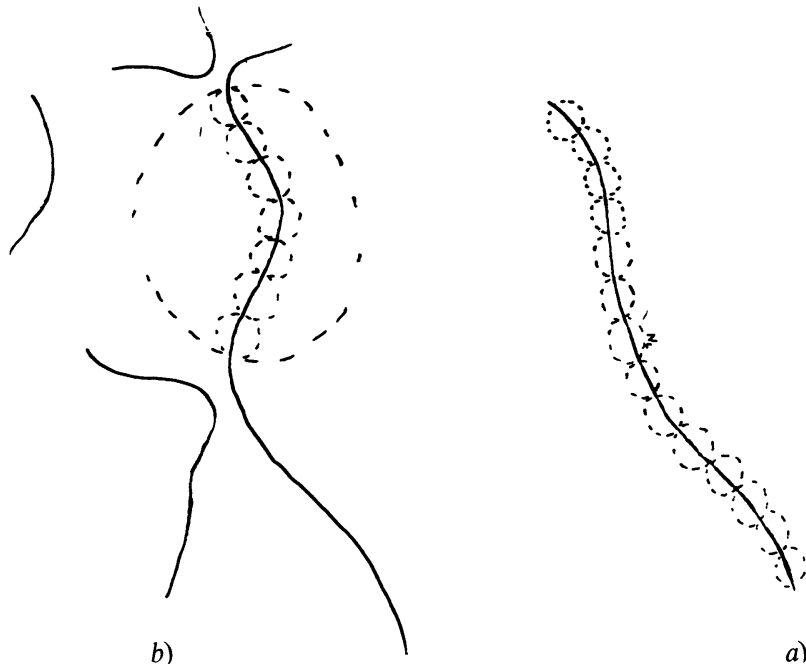

Fig. 1. - - a) The local behaviour of a chain is theta-like. (See notes [10] and [14].) For larger distances, the excluded volume interaction is present. $b$ ) In a semi-dilute solution, inside the blob, there is the same structure as for the single chain.

consider that the discrepancy between the static and dynamic measurements [13] of the radius of the blob is an evidence for this substructure. The same analysis as above can be done for the diffusion of the blob : In order to calculate the diffusion coefficient $D_{\mathrm{c}}$ one has to separate the short distance behaviour (inside the flof) from the larger distance behaviour

$$
D_{\mathrm{c}} \sim \frac{1}{\xi_{\mathrm{H}}}=\frac{1}{2 g^{2}} \sum_{i, j}\left\langle r_{i j}^{-1}\right\rangle
$$

The only difference between (7) and (5) is that one has to replace $N$ by the number $g$ of statistical units in the blob.

$$
D_{\mathrm{c}} \sim \xi_{\mathrm{H}}^{-1}=N_{\tau}^{-1 / 2}\left[\frac{1-2 v}{1-v} \frac{N_{\tau}}{g}+\frac{2 v-1}{3(2-v)}\left(\frac{N_{\tau}}{g}\right)^{2}+\frac{1}{(1-v)(2-v)}\left(\frac{N_{\tau}}{g}\right)^{v}\right]
$$

and we may define an effective exponent by

$$
v_{\mathbf{H}}=\frac{\partial \log \xi_{H}}{\partial \log g} .
$$

Notice that the last term in (8) becomes dominant if $g \gg N_{\tau}$, which is realized if the temperature is very high or the concentration very low. (Usually the limit $v_{\mathrm{H}}=v$ is not achieved.)

Of course this analysis is valid above $C^{*}$ (relation (1)). When the monomer concentration goes to $C^{*}, g$ goes to $N$ and $D$ crosses over smoothly from relation (7) valid in semi-dilute solutions to relation (2) valid in dilute solutions. This can be seen very clearly also in relation (8). So this shows that the cross-over concentration is $C^{*}$ and is the same for the diffusion coefficient as for the radius of gyration. The preceding analysis can be generalized to any other property. One has just to take care of performing the correct dimensional analysis.

An interesting effect is exhibited by this analysis : $g$ depends on both the temperature and the monomer concentration $C$ [15], [16]

$$
g=C^{-5 / 4} \Gamma^{-3 / 4} f\left(\frac{C}{\tau}\right)
$$

where we know the asymptotic behaviour of the function $f(x)$

$$
\begin{array}{ll}
x \rightarrow 0 & f(0)=1 \\
x \gg 1 & f(x) \sim x^{-3 / 4} .
\end{array}
$$


On the other hand $N_{\tau}$ depends on temperature only (relation (6)). So by varying either the temperature at a concentration, or the reverse, one varies the ratio $g / N_{\tau}$, and therefore the value of the exponent $v_{\mathbf{H}}$. We can define effective exponents for the concentration and temperature dependence of $\xi_{\mathbf{H}}$.

$$
\begin{gathered}
\xi_{\mathbf{H}} \sim C^{-\beta} \tau^{-y} \\
\beta=-\frac{\partial \log \xi_{\mathbf{H}}}{\partial \log C} \quad y=\frac{-\partial \log \xi_{\mathbf{H}}}{\partial \log \tau} .
\end{gathered}
$$

Leading to

$$
y=1-\beta
$$

and

$$
\begin{aligned}
& \beta=\frac{\partial \log \xi_{\mathrm{H}}^{-1}}{\partial \log g} \frac{\partial \log g}{\partial \log C} \\
& \beta=v_{\mathrm{H}} \frac{\partial \log g}{\partial \log C} .
\end{aligned}
$$

$v_{\mathrm{H}}$ is easily determined with eqs. (8), and is a decreasing function of $C$. In order to determine the second factor of the right hand side of (11), we may introduce a self-consistent equation for $g$ :

$$
g \sim C \xi^{3}
$$

where $\xi$ is the static radius of the blob :

$$
\begin{aligned}
& \xi^{2}=\frac{1}{N^{2}} \sum_{n}(N-n) r_{n}^{2} \\
& \xi^{2}=\frac{N_{\tau}}{528}\left\{24 x-11 x^{2}+75 x^{-2 v}\right\}
\end{aligned}
$$

where $x=N_{\tau} / g$.

Combining (12) and (13) leads to the self-consistent relation

$\frac{C}{N_{\tau}}=(88)^{3 / 2} x^{-1}\left\{24 x-11 x^{2}+75 x^{-2 v}\right\}^{-3 / 2}$

and from (8), (11) and (14)

$\beta=-\frac{24 x-11 x^{2}+75 x^{-2 v}}{60 x-44 x^{2}-60 x^{-2 v}} \frac{-21 x+4 x^{2}+75 x^{-2 v}}{-21 x+2 x^{2}+75 x^{-2 v}}$

where $x(C / \tau)$ is determined by (14) (we take $N_{\tau} \sim \tau^{-2}$ ).

The corresponding behaviour is shown on figure 2 . The main point we wish to emphasize here is the non monotonic behaviour of the exponent. We discuss this result. The effective exponents are function of $C$ and $\tau$. Some recent light scattering experiments [17] indicate a variation of the exponents with temperature and concentration which may be interpreted this way. Figure 2 shows first a decrease of the exponent with increasing $C$ or decreasing $\tau$. This decrease is due to the competition between the short scale ideal behaviour and the large scale (blob) excluded volume

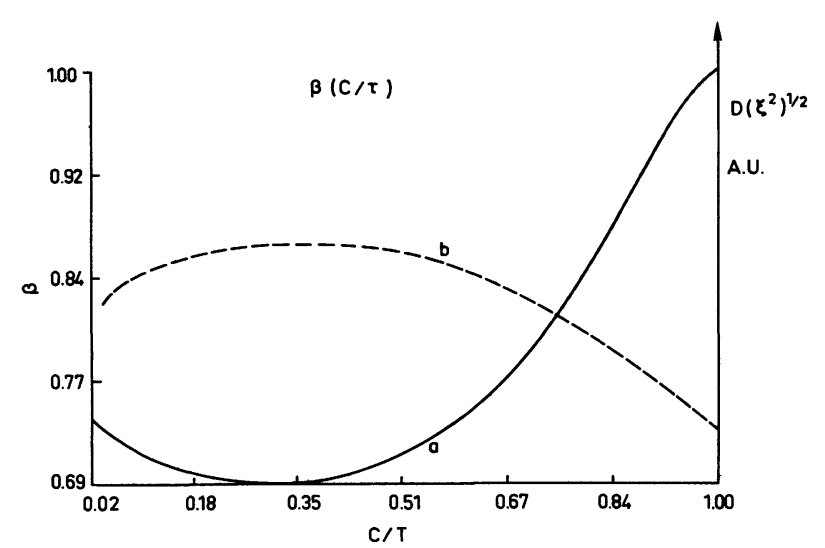

Fig. 2. - a) Variation of the exponent $\beta$ in the diffusion coefficient of the blob $D_{\mathrm{c}} \sim C^{\beta}$ versus $C / \tau$. Note that $C^{*}$ depends on $N$. For very high values of $N, C^{*}$ is very low, and the decreasing part of the curve is easier to observe. $b$ ) Schematic variation of the product $D\left(\xi^{2}\right)^{1 / 2}$ as a function of $C / \tau$.

behaviour. As a result of this competition, the diffusion coefficient depends less strongly on $C$ than in a pure self avoiding walk. When the concentration increases (or the temperature decreases), the size of the blob decreases, and the weight of the flof increases. This is true as long as $g \gg N_{\tau}$. When the concentration is sufficiently high, $g$ itself becomes a rapidly varying function of $C$, see relations (9) and (10), leading to the increase in $\beta$.

The above approach is valid in the good solvent semi-dilute region. Let us discuss briefly the crossovers $C^{*}$ to the dilute regime and $C^{* *}$ to the theta solvent.

i) In the dilute region, the blob becomes the chain itself : There is no dependence on $C$ of the diffusion coefficient. So in the vicinity of $C^{*} \beta$ has to go from equation (15) to the value 0 in the dilute regime. As $C^{*}$ depends on $N$, so does the location of this cross-over. Let us remark here that above this crossover, the experimental data [4] give an exponent of the order of 0.69 showing indeed a decrease. It would be interesting to go both to higher and lower molecular weights.

The higher molecular weights should exhibit the decreasing part of the curve figure 2 .

The lower molecular weights are also interesting because they can tell us how low the effective exponent can actually be.

ii) For concentrated solutions, the chain becomes roughly ideal at every scale. So we expect the exponent to have the asymptotic value 1 in this region. A weakness of the above approach is that the exponent reaches its asymptotic limit at $C^{* *} \sim \tau$ whereas we know that this is just a cross-over concentration, and that the exponent reaches its asymptotic limit for higher values of $C / \tau$. This is due to our model, where we suppose a clear cut between gaussian and excluded volume behaviours. In order to avoid this one has to introduce a more realistic cross-over function for $r_{i j}$. 
Let us finally remark that relation (15) indicate that the variation of the exponent with $C / \tau$ is universal for $C \gg C^{*}$ (in terms of $\xi_{\mathrm{H}}$ or $D_{\mathrm{c}}$ ), the coordinates $C^{-1} \xi_{\mathrm{H}}$ or $D_{\mathrm{c}} C$ vs. $C / \tau$ are the most appropriate. Moreover, as we are dealing with local properties, the polydispersity is irrelevant. As a conclusion, we extended the blob approach to the diffusion of the blob in semi-dilute solutions. We showed that the cross-over concentration $C^{*}$ is the same for static and dynamic properties indeed, when the correct dimensional analysis is performed. For semi-dilute solutions, we could define an effective exponent for the variation of the diffusion coefficient of the blob with concentration. This exponent has a non monotonous behaviour with increasing $C$ or decreasing $\tau$. In particular, it should decrease in the semi-dilute regime. This effect is due to the competition between the short scale ideal behaviour and the large scale (blob) excluded volume behaviour. It should be observed by plotting experimental values of the product $D\left(\xi^{2}\right)^{1 / 2}$ against $C / \tau$ (see Fig. $2 b$ ). Diffusion coefficients $D$ are obtained from the quasielastic broadening of an incident light beam, and the blob squared length $\xi^{2}$ is measured from the neutron scattering experiment.

Acknowledgments. - The authors wish to thank G. Benedek, S. Redner, H. E. Stanley and T. Witten for related discussions, E. Geissler and A. M. Hecht for a very stimulating correspondence, and one of our referees for very valuable comments. This work was partly supported by a grant of A.F.O.S.R.

\section{References}

[1] Weill, G., Des Cloizeaux, J., J. Physique 40 (1979) 99.

[2] Axcasu, A. Z., HaN, C. C., Macromolecules, in press.

[3] FloRy, P. J., Principles of Polymer Chemistry (Cornell) 1953.

[4] Adam, M., Delsanti, M., Jannink, G., J. Physique Lett. 37 (1976) L-53.

Delsanti, M., Thèse, Université Paris-Sud Orsay. Available at C.E.N. Saclay, S.P.S.R.M.

Munch, J. P., Candau, S., Herz, J., Hild, G., J. Physique 38 (1977) 971 .

[5] Stockmayer, W. H., Albrecht, A. C., J. Polym. Sci. 32 (1958) 215.

[6] De Gennes, P. G., Phys. Lett. 38A (1972) 339.

[7] Stockmayer, W. H., in Molecular Fluids, les Houches Summer School, Balian, R. and Weill, G., ed. (1973).

[8] Muthuxumar, M., Freed, K. F., Macromolecules 10 (1977) 899.
[9] Ferry, J. D., Viscoelastic properties of Polymers, J. Wiley ed. (1961).

[10] See for instance Farnoux, B. et al., J. Physique 39 (1978) 77. See also note [14].

[11] For low concentrations, the analysis holds for $\eta$.

[12] DaOud, M. et al., Macromolecules 8 (1975) 804.

[13] As in the dilute case, the laws for $\xi$ are not the same by static [12] or dynamic [4] experiments, and one can also define $\xi_{\mathrm{H}}$ in the same way as in the dilute regime.

[14] In this respect, the substructure in the single chain should have the same denomination, to distinguish it from the blob which was initially introduced for semi-dilute solutions (see ref. [12]).

[15] Daoud, M., Thèse, Université Paris VI (1977).

[16] Cotron, J. P. et al., J. Chem. Phys. 65 (1976) 76.

[17] Geissler, E. and HeCht, A. M., J. Physique Lett. 40 (1979) L-173 and private communication. 